\title{
PARA ALÉM DA ILITERACIA EM SAÚdE: QUESTÕES E REAÇÕES
}

\author{
Pedro de Matos Gonçalves \\ Politécnico de Leiria \\ pjmatosg@ipleiria.pt \\ Susana Mendes \\ Politécnico de Leiria \\ susana.mendes@ipleiria.pt \\ Sílvio Manuel Brito \\ Instituto Politécnico de Tomar \\ Silvio.brito@ipt.pt \\ Orlindo Gouveia Pereira \\ Professor Emérito \\ o.pra@me.com
}

Recepción Artículo: 13 mayo 2021

Admisión Evaluación: 13 mayo 2021

Informe Evaluador 1: 16 mayo 2021

Informe Evaluador 2: 18 mayo 2021

Aprobación Publicación: 01 junio 2021

\section{RESUMO}

Em situações catastróficas como a que estamos a viver cresce na população um sentimento de alarme que altera o modo de encarar a vida do dia-a-dia com um futuro incerto, inclusive a própria saúde. Criam-se mesmo novos hábitos de pensar e de agir. Se examinarmos esta questão no plano interpessoal da Psicologia Social deparamo-nos com dissonâncias afetivas e cognitivas que podem chegar ao "negacionismo" no que respeita às medidas preventivas decretadas pelo Governo, à toma das vacinas e medicamentos, e à apreciação do próprio estado de saúde.

Levados por uma moda que custa a passar, alguns tentam explicar estas atitudes e comportamentos invocando a iliteracia em saúde. Em estudos anteriores 0.G.Pereira, P.M. Gonçalves, S. Brito e S. Mendes (2014; 2016), mostraram que a iliteracia é um fator pouco relevante, sendo de atribuir os efeitos encontrados ao stresse, às estratégias de coping, e aos hábitos e estilos de vida nocivos.

0 presente estudo longitudinal visa aprofundar os resultados anteriores, utilizando instrumentos semelhantes, mas tendo em consideração o que aconteceu aos mesmos estudantes-sujeitos nos últimos três anos, sendo o último já o da Pandemia.

Os dados foram estudados por ano e por escola (Saúde e Turismo e Tecnologia Marítima). Os resultados evidenciaram que o estado de saúde não varia entre os anos e as escolas. Além disso, 


\section{PARA ALÉM DA ILITERACIA EM SAÚdE: QUESTÕES E REAÇ̃̃ES}

foi significativo que as estratégias de coping associadas ao pensamento levaram à negação ou ao uso do humor para superar o problema.

Os dados antropométricos são considerados em seis gráficos, por ano e escola. A avaliação da saúde é apresentada na tabela 1. As inter-relações entre as estratégias de enfrentamento (ação e pensamento) com os hábitos nocivos à saúde estão nas tabelas 2, 3, 4 e 5.

Palavras chave: saúde; stresse; estratégias de enfrentamento (coping); iliteracia; estudantes de politécnico

\section{ABSTRACT}

Beyon health illiteracy - questions and reactions. In catastrophic situations such as the one we are experiencing, because of COVID-19 pandemic, a feeling of alarm grows in the population and alters the usual ways of facing uncertain future, including our own health. New habits of thinking and acting are emerging.

At the interpersonal level of Social Psychology, we are faced with affective and cognitive dissonances that can reach "negationism" with regard to the preventive measures decreed by the Government, the taking of vaccines and medications, and the assessment of one s own health.

Driven by a fad that is hard to pass, some try to explain these attitudes and behaviors by invoking illiteracy in health. In previous studies, O.G. Pereira, P.M. Gonçalves, S. Brito and S. Mendes (2014; 2016), showed that illiteracy is a minor factor. It is better to attribute the effects found to stress, coping strategies, and harmful habits and life styles, if you postulate a circular causality among them.

The present longitudinal study aims to deepen the previous findings, using similar instruments, but taking into account what happened to the same student-subjects in the last three years, the last one being that of the Pandemic. Data were studied by year and school (Health Sciences and Tourism and Maritime Technology).

The results showed that health evaluation do not varies across years and schools. In addition, it was significant that the coping strategies of thought led to denial or to the use of humor to get past the problem. Anthropometric data are considered in six graphics, by year and school. Health evaluation are presented in table 1. The interrelations among coping strategies (action and though) with noxious health habits are in tables 2, 3, 4 and 5.

Keywords: health; stress; coping strategies; illiteracy; polytechnic students

\section{INTRODUÇÃO}

0 presente estudo resulta de um insólito encontro entre "necessidade e acaso". Prosseguíamos uma investigação, que tem como sujeitos estudantes do Politécnico de Leira, no domínio psicossociológico da saúde que começou por questionar se a iliteracia em Saúde explica os níveis habituais de stresse e a persistência e aquisição de hábitos nocivos nutricionais e de dependência: estava em curso a observação longitudinal de estudantes de duas escolas - de saúde e de turismo - ao longo de três anos. Aconteceu que o terceiro ano foi de confinamento e de outras alterações da vida académica por força da pandemia COVID-19.

0 primeiro estudo (Pereira, O.G., Gonçalves, P., 2014 e Gonçalves, P., Brito, S., Pereira, O.G. \& Ravara, L., 2014) realizou um inquérito a 196 estudantes do Politécnico de Leiria. Como grupo de controlo foram considerados 739 sujeitos com habilitações superiores e 98 sujeitos toxicodependentes (estes por entrevista). Os resultados alcançados permitiram concluir que os níveis habituais de stresse e a persistência de hábitos nocivos para a saúde são mais determinantes que a iliteracia em saúde e a dependência de substâncias, nos efeitos considerados. A partir deste estudo passámos a centrar a atenção nos hábitos nutricionais e da vida comum dos estudantes. Utilizar estu- 
dantes de uma escola de saúde em confronto com uma escola de turismo passou a servir para, por um lado, avaliar o efeito da educação sanitária continuada e, por outro, outros estilos de vida académica.

Em sequência (Gonçalves, P, Pereira, O.G, Mendes, S., 2016) procurámos verificar como os sujeitos faziam autoavaliações da própria saúde e dos hábitos deletérios para ela e determinar 0 papel das estratégias de enfrentamento - coping - que servem a adaptação dos sujeitos ao stresse. Concluímos que é necessário ir à raiz das atitudes, hábitos e disposições comportamentais para estruturar intervenções sanitárias. Neste sentido, foi possível observar que 0 fator literacia não é relevante.

Na investigação seguinte (Pereira, 0.G., Gonçalves. P., Mendes, S., 2016), com 290 sujeitos determinou-se que as estratégias de enfrentamento - coping - divididas em "de pensamento" e "de ação", modelam os efeitos do stresse na manutenção de hábitos nefastos para a saúde num circuito de causalidade circular. Os sujeitos não estão conscientes destes efeitos ou respondem dissonantemente, com "atribuições externas". Daqui se partiu para o estudo de medidas que tenham em consideração estes circuitos de causalidade circular. Para o fazer tivemos de utilizar o modelo longitudinal: observar os estudantes que entram nas duas escolas selecionadas do Politécnico, à entrada e nos dois anos seguintes. De salientar que, o terceiro foi 0 da pandemia.

Os estudantes que vêm frequentar o Politécnico, sobretudo os que vêm de locais distantes da sede das escolas (respetivamente, Leiria e Peniche) estão particularmente sujeitos a stresse e, hipoteticamente, a desenvolverem estratégias de enfrentamento - coping - diferentes dos que continuam a viver em suas casas. A pandemia com o confinamento elevou o nível do stresse e muito provavelmente levou a mudanças organísmicas (como por ex.: engordar ou emagrecer) e das estratégias habituais de enfrentamento - coping.

Temos em atenção que algo de semelhante foi referido no relatório de 2019 da National Academies of Sciences, Engineering and Medicine dos EUA (Denworth., L., 2021). Naqueles que antigamente eram chamados adolescentes, isto é, dos 13 aos 24 anos, reconheceu-se a importância da secreção de testosterona (nos dois sexos), que contribui para o nível de stresse e controla 0 desenvolvimento corporal, facilitando as estratégias de enfrentamento - coping - de ação.

\section{MÉTODO}

\section{Sujeitos}

Da amostra total de 189 sujeitos, os 83 do ano letivo 2018-2019 sofreram atrição e apenas 35 estudantes responderam ao questionário no ano letivo de 2020-2021.

\section{Instrumento}

0 questionário é adaptado dos sucessivamente utilizados nos estudos anteriores. Inclui questões de resposta simples ou múltipla sobre o estado de saúde atual, toma de medicamentos, hábitos alimentares, uso de bebidas alcoólicas, drogas ilícitas, fumar e atividade física. Complementarmente eram colhidos dados antropométricos: idade, sexo, altura, peso, perímetro abdominal e local de residência.

No questionário faziam-se perguntas diretas sobre comportamentos dos sujeitos, tanto de resposta "sim" ou "não", ou de cinco posições ordinais (ex: estado de saúde: "muito bom", "bom", "razoável", "mau" e "muito mau" / bebidas alcoólicas: "nunca", "esporadicamente", "às refeições", "saídas à noite").

As questões relativas a alimentos contendo açúcar ou contendo sal (fast food) eram tabeladas separadamente com as seguintes posições ordinais (ex: "nunca", "1x dia", "1x semana", "mais que uma vez por semana", "esporadicamente"). 


\section{PARA ALÉM DA ILITERACIA EM SAÚdE: QUESTÕES E REAÇ̃̃ES}

As 12 questões relativas às estratégias de enfrentamento - coping - eram fechadas e de teor semelhante. Exemplos:

1 - "Quando me apontam ou detetam um problema nos hábitos alimentares faço esforços para os mudar".

9 - "Quando me apontam ou detetam um problema nos hábitos alimentares costumo dizer a mim próprio "isto não é verdade".

Em qualquer dos casos a escala de resposta utilizada foi de "nunca", "quase nunca", "às vezes", "com alguma frequência" e "muito frequentemente".

A questão 1 é codificada como estratégia de ação e a questão 9 é codificada como estratégia de pensamento (há mais cinco semelhantes de cada no questionário).

\section{ANÁLISE ESTATÍSTICA}

Os dados quantitativos e qualitativos foram estudados e os resultados são apresentados com recurso a tabelas, quadros e gráficos, elaborados especificamente para cada situação em análise. Para a avaliação das variáveis antropométricas (peso, perímetro abdominal e índice de massa corporal), quando comparados os anos letivos e as escolas, recorreu-se à análise de variância (ANOVA) com um fator. Todos os pressupostos (nomeadamente, normalidade dos dados e homogeneidade de variâncias) foram validados. Sempre estes não foram cumpridos recorreu-se ao teste não-paramétrico de Kruskal-Wallis (Zar, J.H., 2010). Sempre que aplicável, forma realizados testes de comparações múltiplas, nomeadamente o teste de Tukey HSD (no caso de os pressupostos da ANOVA serem cumpridos) ou de Games-Howell (no caso de os pressupostos da ANOVA não serem cumpridos) (Zar, J.H., 2010). Para a análise das variáveis da Saúde recorreu-se ao teste não-paramétrico de independência do Qui-Quadrado (Siegel, S., 1988; Swinscow, T.D., Campbell, M.J., 2002). Este é um teste baseado na construção de tabelas de contingência e permite verificar a independência entre duas variáveis que, sendo expressas em qualquer escala se apresentam em classes mutuamente exclusivas e exaustivas. 0 teste do Qui-Quadrado é suficientemente robusto quanto aos pressupostos que impõe e adequado para o estudo de variáveis de natureza qualitativa, pelo que justifica a sua aplicação nos dados em análise (Siegel, S., 1988). Sempre que os pressupostos não foram cumpridos, recorreu-se ao teste exato de Fisher-Freeman-Halton (Mehta, C.R., Patel N.R., 1983). Todos os resultados foram considerados estatisticamente significativos ao nível de $5 \%$ (ou seja, sempre que $\mathrm{p}$-value $<0,05)$. Todos os cálculos forma realizados com recurso ao software IBM SPSS Statistics 27 (Copyright IBM Corp. (1989-2019, Armonk, New York 10504-1722, USA).

\section{RESULTADOS}

A análise longitudinal das variáveis antropométricas revelou:

\section{Peso, Perímetro Abdominal e Índice de Massa corporal (IMC) - Escola de Saúde}

0 peso e o perímetro abdominal mantêm-se estáveis no $1^{0}$ e $2^{\circ}$ anos, sendo que no $3^{\circ}$ ano (em que houve confinamento) apresenta uma diminuição evidente (Figs. 1(a), (b)). No que respeita ao IMC, este aumentou do $1^{\circ}$ para $02^{\circ}$ ano, tendo diminuído no $3^{\circ}$ para um nível igual ao do $1^{\circ}$ (Fig. (c)). Contudo, as diferenças não se evidenciaram como sendo estatisticamente significativas $(p>0,05)$ para nenhumas das situações em análise. 
Figura 1

Variação das variáveis antropométricas ao longo dos três anos letivos (2018-19, 2019-20 e 2020-21), para a escola de Saúde (ESSLei)
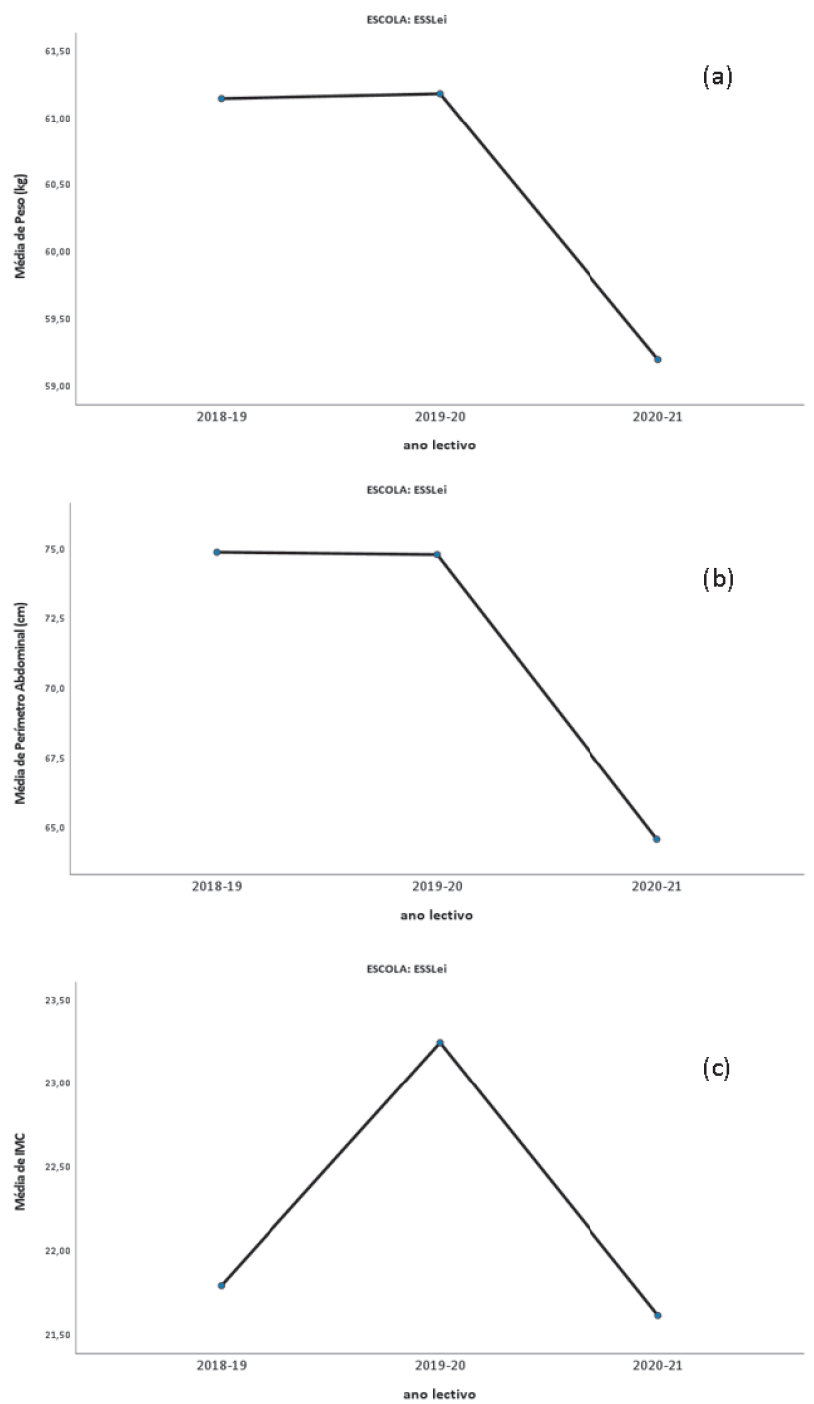

Peso, Perímetro Abdominal e Índice de Massa corporal (IMC) - Escola Turismo

0 peso e o perímetro abdominal apresentam padrão similar, mantendo-se estáveis no $1^{0}$ e $2^{0}$ anos, mas evidenciando um aumento no caso do $3^{0}$ ano (Figs. 2(a) e (b)). Relativamente ao IMC observou-se uma evolução de diminuição ao longo dos três anos em análise (Fig. 2(c)). Todavia, de 


\section{PARA ALÉM DA ILITERACIA EM SAÚdE: QUESTÕES E REAÇÕES}

forma similar ao padrão anterior, os resultados não evidenciaram diferenças estatisticamente significativas $(p>0,05)$.

\section{Figura 2}

Variação das variáveis antropométricas ao longo dos três anos letivos (2018-19, 2019-20 e 2020-21), para a escola de Turismo (ESTM)
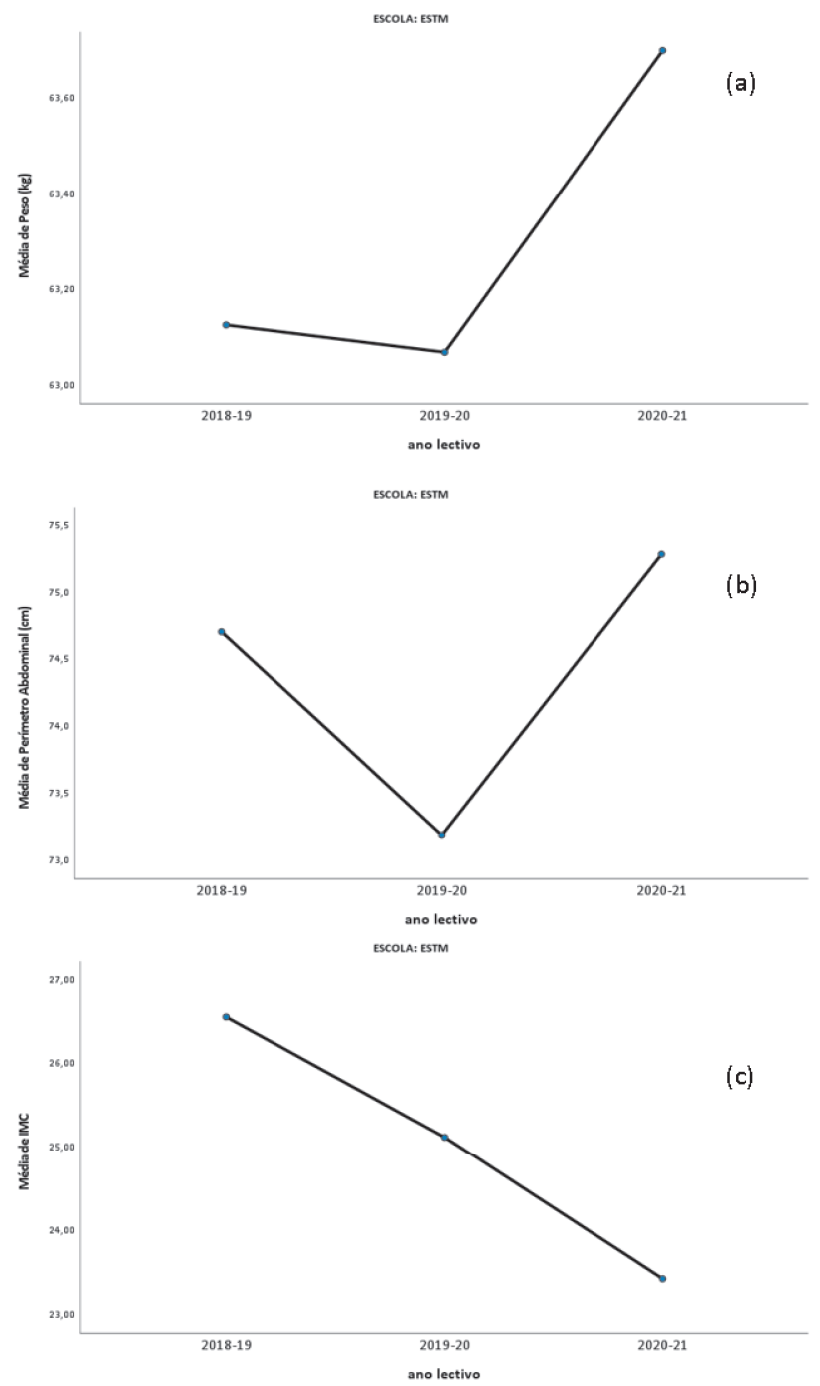

Em suma, e centrando a análise no $3^{0}$ ano, os resultados alcançados evidenciaram que os estudantes da escola de Saúde apresentam uma diminuição no peso, perímetro abdominal e IMC. Ao invés, os estudantes da escola de Turismo apresentam um aumento de peso que acompanha o perí- 
metro abdominal, sendo que o IMC diminui. De salientar que o IMC tem um padrão similar (de diminuição) em ambos os casos.

\section{Variáveis de Saúde}

A análise das variáveis de saúde revela que o panorama é quase sobreponível nas duas escolas no $3^{0}$ ano de observação (Tabela 1).

Tabela 1

Distribuição das frequências para as variáveis do estado de saúde no conjunto das duas escolas $(n=35)$.

\begin{tabular}{|c|c|c|c|}
\hline & Saúde & Turismo & Total \\
\hline Muito bom & 9 & 6 & 15 \\
\hline Bom & 26 & 26 & 52 \\
\hline Razoável & 5 & 14 & 19 \\
\hline Mau & 0 & 0 & 0 \\
\hline
\end{tabular}

\section{Estratégias de Coping}

Os resultados alcançados demonstraram que existem variações nas estratégias de ação diferenciadas por escola.

Nas tabelas 2 a 5 os resultados são baseados em linhas e colunas não vazias em cada subtabela mais interna.

Na escola de saúde (ESSLei) há uma múltipla influência significativa entre consumo de "bebidas alcoólicas" e "amigos e conhecidos".

Tabela 2

Estratégias de coping (ESSLei) (n=35).

\begin{tabular}{|c|c|c|c|c|c|c|c|}
\hline \multicolumn{8}{|c|}{ Pearson chi square tests } \\
\hline & & $\begin{array}{l}\text { [Action] } \\
\text { Change }\end{array}$ & $\begin{array}{c}\text { [Action] } \\
\text { Information }\end{array}$ & $\begin{array}{c}\text { [Action] } \\
\text { Health } \\
\text { Professional }\end{array}$ & $\begin{array}{c}\text { [Action] } \\
\text { Friends and } \\
\text { acquaintances }\end{array}$ & $\begin{array}{l}\text { [Action] } \\
\text { Search for } \\
\text { something }\end{array}$ & $\begin{array}{c}\text { [Action] Doing } \\
\text { other things }\end{array}$ \\
\hline \multirow[t]{3}{*}{ Health condition } & Chi-square & 4,417 & 2,914 & 7,700 & 9,708 & 8,792 & 8,250 \\
\hline & gl & 6 & 4 & 8 & 6 & 8 & 6 \\
\hline & Sig. & 0,62 & 0,572 & 0,463 & 0,137 & 0,36 & 0,22 \\
\hline \multirow[t]{3}{*}{ Sugar in drinks } & Chi-square & 1,111 & 1,746 & 5,625 & 2,153 & 5,625 & 2,500 \\
\hline & gl & 3 & 2 & 4 & 3 & 4 & 3 \\
\hline & Sig. & 0,774 & 0,418 & 0,229 & 0,541 & 0,229 & 0,475 \\
\hline \multirow[t]{3}{*}{ Salt in meals } & Chi-square & 0,750 & 0,171 & 6,900 & 4,500 & 8,250 & 2,625 \\
\hline & gl & 3 & 2 & 4 & 3 & 4 & 3 \\
\hline & Sig. & 0,861 & 0,918 & 0,141 & 0,212 & 0,083 & 0,453 \\
\hline \multirow[t]{3}{*}{ water between meals } & Chi-square & & & & & & \\
\hline & gl & --- & --- & --- & --- & --- & --- \\
\hline & Sig. & & & & & & \\
\hline \multirow{3}{*}{$\begin{array}{l}\text { Alcoholic beverages } \\
\text { consumption frequency }\end{array}$} & Chi-square & 11,875 & 8,200 & 5,513 & 16,438 & 6,313 & 3,563 \\
\hline & gl & 6 & 4 & 8 & 6 & 8 & 6 \\
\hline & Sig. & 0,065 & 0,085 & 0,702 &, $012^{*}$ & 0,612 & 0,736 \\
\hline \multirow[t]{3}{*}{ Smoking habits } & Chi-square & 0,577 & 2,637 & 4,183 & 2,019 & 3,462 & 1,587 \\
\hline & gl & 3 & 2 & 4 & 3 & 4 & 3 \\
\hline & Sig. & 0,902 & 0,267 & 0,382 & 0,568 & 0,484 & 0,662 \\
\hline
\end{tabular}

* diferenças estatisticamente significativas ao nível de 5\%.

Na escola de turismo (ESTM) deteta-se o mesmo estado de relações significativas entre "refeições salgadas" e "doces". 0 "hábito de fumar" corresponde, significativamente, a "necessidade de mudança". 
PARA ALÉM DA ILITERACIA EM SAÚdE: QUESTÕES E REAÇÕES

Tabela 3

Estratégias de coping (ESTM) (n=35)

\begin{tabular}{|c|c|c|c|c|c|c|c|}
\hline \multicolumn{8}{|c|}{ Pearson chi square tests } \\
\hline & & $\begin{array}{l}\text { [Action] } \\
\text { Change }\end{array}$ & $\begin{array}{c}\text { [Action] } \\
\text { Information }\end{array}$ & $\begin{array}{c}\text { [Action] } \\
\text { Health } \\
\text { Professional }\end{array}$ & $\begin{array}{c}\text { [Action] } \\
\text { Friends and } \\
\text { acquaintances }\end{array}$ & $\begin{array}{l}\text { [Action] } \\
\text { Search for } \\
\text { something }\end{array}$ & $\begin{array}{l}\text { [Action] Doing } \\
\text { other things }\end{array}$ \\
\hline \multirow[t]{3}{*}{ Health condition } & Chi-square & 9,020 & 4,476 & 7,767 & 5,667 & 5,024 & 7,500 \\
\hline & $\mathrm{gl}$ & 6 & 6 & 8 & 8 & 6 & 4 \\
\hline & Sig. & 0,172 & 0,613 & 0,457 & 0,685 & 0,541 & 0,112 \\
\hline \multirow[t]{3}{*}{ Sugar in drinks } & Chi-square & 6,667 & 8,357 & 3,015 & 5,850 & 1,270 & 5,538 \\
\hline & $\mathrm{gl}$ & 3 & 3 & 4 & 4 & 3 & 2 \\
\hline & Sig. & 0,083 & $039^{*}$ & 0,555 & 0,211 & 0,736 & 0,063 \\
\hline \multirow[t]{3}{*}{ Salt in meals } & Chi-square & 10,850 & 4,500 & 4,500 & 7,875 & 1,728 & 1,904 \\
\hline & $\mathrm{gl}$ & 3 & 3 & 4 & 4 & 3 & 2 \\
\hline & Sig. &, $013^{*}$ & 0,212 & 0,343 & 0,096 & 0,631 & 0,386 \\
\hline \multirow[t]{3}{*}{ water between meals } & Chi-square & 5,965 & & & & & \\
\hline & $\mathrm{gl}$ & 3 & --- & -- & --- & --- & --- \\
\hline & Sig. & 0,113 & & & & & \\
\hline \multirow{3}{*}{$\begin{array}{l}\text { Alcoholic beverages } \\
\text { consumption frequency }\end{array}$} & Chi-square & 5,148 & 7,052 & 3,042 & 9,563 & 8,720 & 9,436 \\
\hline & $\mathrm{g} \mid$ & 6 & 6 & 8 & 8 & 6 & 4 \\
\hline & Sig. & 0,525 & 0,316 & 0,932 & 0,297 & 0,19 & 0,051 \\
\hline \multirow[t]{3}{*}{ Smoking habits } & Chi-square & 10,044 & 4,555 & 2,764 & 4,500 & 4,018 & 1,755 \\
\hline & gl & 3 & 3 & 4 & 4 & 3 & 2 \\
\hline & Sig. & $018^{*}$ & 0,207 & 0,598 & 0,343 & 0,26 & 0,416 \\
\hline
\end{tabular}

* diferenças estatisticamente significativas ao nível de 5\%.

A nível das estratégias de pensamento na Escola de saúde (ESSLei) dizer a si próprio que "não é verdade" está, significativamente, relacionado com o abuso "do açúcar em bebidas". Por outro lado, 0 uso do humor corresponde ao "consumo frequente de bebidas alcoólicas" e também é significativo.

\section{Tabela 4}

Estratégias de coping (ESSLei) ( $n=35)$.

\begin{tabular}{|c|c|c|c|c|c|c|c|}
\hline \multicolumn{8}{|c|}{ Pearson chi square tests } \\
\hline & & $\begin{array}{c}\text { [Thinking] I do } \\
\text { not care }\end{array}$ & $\begin{array}{l}\text { [Thinking] } \\
\text { Planning }\end{array}$ & $\begin{array}{l}\text { [Thinking] } \\
\text { worried }\end{array}$ & $\begin{array}{c}\text { [Thinking] "It's } \\
\text { not true" }\end{array}$ & $\begin{array}{c}\text { [Thinking] } \\
\text { Humour }\end{array}$ & $\begin{array}{l}\text { [Thinking] } \\
\text { Scientific } \\
\text { question }\end{array}$ \\
\hline \multirow[t]{3}{*}{ Health condition } & Chi-square & 4,955 & 8,675 & 9,986 & 5,356 & 5,063 & 9,000 \\
\hline & gl & 2 & 8 & 6 & 4 & 4 & 8 \\
\hline & Sig. & 0,084 & 0,37 & 0,125 & 0,253 & 0,281 & 0,342 \\
\hline \multirow[t]{3}{*}{ Sugar in drinks } & Chi-square & 0,045 & 4,097 & 5,774 & 7,963 & 2,847 & 3,889 \\
\hline & $\mathrm{gl}$ & 1 & 4 & 3 & 2 & 2 & 4 \\
\hline & Sig. & 0,833 & 0,393 & 0,123 & $019^{*}$ & 0,241 & 0,421 \\
\hline \multirow[t]{3}{*}{ Salt in meals } & Chi-square & 0,134 & 2,775 & 3,171 & 2,600 & 0,188 & 7,125 \\
\hline & $\mathrm{gl}$ & 1 & 4 & 3 & 2 & 2 & 4 \\
\hline & Sig. & 0,714 & 0,596 & 0,366 & 0,273 & 0,911 & 0,129 \\
\hline \multirow[t]{3}{*}{ water between meals } & Chi-square & & & & & & \\
\hline & $\mathrm{gl}$ & --- & -- & --- & --- & --- & --- \\
\hline & Sig. & & & & & & \\
\hline \multirow{3}{*}{$\begin{array}{l}\text { Alcoholic beverages } \\
\text { consumption frequency }\end{array}$} & Chi-square & 3,549 & 7,825 & 2,389 & 2,700 & 12,250 & 13,875 \\
\hline & gl & 2 & 8 & 6 & 4 & 4 & 8 \\
\hline & Sig. & 0,17 & 0,451 & 0,881 & 0,609 &, $016^{*}$ & 0,085 \\
\hline \multirow[t]{3}{*}{ Smoking habits } & Chi-square & 0,010 & 4,904 & 2,637 & 0,385 & 0,938 & 2,740 \\
\hline & $\mathrm{gl}$ & 1 & 4 & 3 & 2 & 2 & 4 \\
\hline & Sig. & 0,919 & 0,297 & 0,451 & 0,825 & 0,626 & 0,602 \\
\hline
\end{tabular}

* diferenças estatisticamente significativas ao nível de 5\%.

Na escola de turismo (ESTM) a relação significativa é entre o abuso de "sal nas refeições" e a estratégia "não me importo". 
Tabela 5

Estratégias de coping (ESTM) ( $n=35)$

\begin{tabular}{|c|c|c|c|c|c|c|c|}
\hline \multicolumn{8}{|c|}{ Pearson chi square tests } \\
\hline & & $\begin{array}{c}\text { [Thinking] I do } \\
\text { not care }\end{array}$ & $\begin{array}{l}\text { [Thinking] } \\
\text { Planning }\end{array}$ & $\begin{array}{l}\text { [Thinking] } \\
\text { worried }\end{array}$ & $\begin{array}{c}\text { [Thinking] "It's } \\
\text { not true" }\end{array}$ & $\begin{array}{l}\text { [Thinking] } \\
\text { Humour }\end{array}$ & $\begin{array}{l}\text { [Thinking] } \\
\text { Scientific } \\
\text { question }\end{array}$ \\
\hline \multirow[t]{3}{*}{ Health condition } & Chi-square & 2,800 & 8,000 & 7,855 & 6,952 & 1,848 & 10,967 \\
\hline & gl & 6 & 8 & 6 & 4 & 4 & 8 \\
\hline & Sig. & 0,833 & 0,433 & 0,249 & 0,138 & 0,764 & 0,204 \\
\hline \multirow[t]{3}{*}{ Sugar in drinks } & Chi-square & 1,800 & 5,040 & 4,451 & 1,125 & 0,205 & 4,095 \\
\hline & gl & 3 & 4 & 3 & 2 & 2 & 4 \\
\hline & Sig. & 0,615 & 0,283 & 0,217 & 0,57 & 0,903 & 0,393 \\
\hline \multirow[t]{3}{*}{ Salt in meals } & Chi-square & 7,875 & 4,500 & 0,695 & 1,848 & 3,656 & 3,150 \\
\hline & gl & 3 & 4 & 3 & 2 & 2 & 4 \\
\hline & Sig. &, $049^{*}$ & 0,343 & 0,874 & 0,397 & 0,161 & 0,533 \\
\hline \multirow[t]{3}{*}{ water between meals } & Chi-square & & & & & & \\
\hline & gl & --- & --- & --- & --- & --- & --- \\
\hline & Sig. & & & & & & \\
\hline \multirow{3}{*}{$\begin{array}{l}\text { Alcoholic beverages } \\
\text { consumption frequency }\end{array}$} & Chi-square & 11,550 & 13,392 & 4,909 & 1,738 & 6,388 & 8,567 \\
\hline & gl & 6 & 8 & 6 & 4 & 4 & 8 \\
\hline & Sig. & 0,073 & 0,099 & 0,556 & 0,784 & 0,172 & 0,38 \\
\hline \multirow[t]{3}{*}{ Smoking habits } & Chi-square & 1,511 & 4,693 & 3,904 & 0,505 & 3,097 & 3,343 \\
\hline & gl & 3 & 4 & 3 & 2 & 2 & 4 \\
\hline & Sig. & 0,68 & 0,32 & 0,272 & 0,777 & 0,213 & 0,502 \\
\hline
\end{tabular}

* diferenças estatisticamente significativas ao nível de 5\%.

\section{DISCUSSÃO}

Observaram-se variações nos dados antropométricos por escolas, que demonstram o efeito direto do confinamento e outras alterações das condições de vida académica, familiar e social (como por exemplo mudar de habitação), no estado de stresse dos estudantes ao longo do último ano.

Por um lado, este stresse crónico não alterou a perceção do próprio estado de saúde reportado pelos sujeitos, mas teve alguma influência em mudanças nas estratégias de coping, tanto nas de ação como nas de pensamento.

Se a apreciação do próprio "estado de saúde" não teve variação, nem no tempo nem entre as escolas, estiveram em causa mudança para hábitos alimentares deletérios, como refeições com excesso de sal ou de açúcar, o consumo de bebidas alcoólicas e fumar. É significativo que as estratégias de coping de pensamento levaram há negação ou ao uso do humor para passar ao lado deste problema. Pode sustentar-se que esta é a via do negacionismo (que pululou na sociedade mais vasta, que sofreu o confinamento e outras restrições).

Embora fosse reconhecida "a necessidade de mudança" as estratégias mais usadas foram 0 "humor" e procurar estar com os "amigos" e "conhecidos", afastando assim ocupar-se com o problema vivido. É um recuso a uma forma de "repressão" que se revela eficiente.

Neste estudo não se consideraram medidas de stresse e tomou-se como possível indicador de literacia a divisão dos estudantes pelas escolas consideradas, com base, nas investigações anteriormente realizadas. No entanto, as variações dos dados antropométricos, bem como as estratégias de coping significativas detetadas, têm muito provavelmente origem nele. Saliente-se finalmente a eficácia da perspetiva psicossocial nestes domínios.

\section{REFERÊNCIAS BIBLIOGRÁFICAS}

Denworth., L. (2021). Age of Opportunity. Sicentific American, 324, 5. (52-57).

Gonçalves, P., Brito, S., Pereira, O.G. \& Ravara, L. (2014). "Superar a lliteracia em Saúde". IX Congresso Iberoamericano de Psicologia/2 Congresso da OPP. 


\section{PARA ALÉM DA ILITERACIA EM SAÚDE: QUESTÕES E REAÇÕES}

Gonçalves, P., Brito, S., Pereira, O.G., Ravara, L. (2015): "Para Além da Iliteracia em Saúde". Psicologia na Atualidade, 27, 44-59.

Gonçalves, P, Pereira, 0.G, Mendes, S. (2016): "O Papel de stresse e de coping nas doenças comportamentais" In Atas do $3^{0}$ Congresso da Ordem dos Psicólogos Portugueses, Lisboa, 28 setembro 2016 a 1 de outubro 2016 (pp. 680-687). Lisboa

Pereira, 0.G.; Gonçalves, P. (2014): "Health illiteracy in students of a polytechnic institute". In $2^{\circ}$ Congresso Internacional de Saúde: Challenges \& Innovation in health, Leiria 9 e 10 maio (pp. 111). Revista de Saúde Publica. São Paulo-Brasil.

Pereira, O.G., Gonçalves. P., Mendes, S. (2016): "Promoção da Saúde por via da modificação de estilos de vida e hábitos nefastos". In Livro de resumos do IX Simpósio Nacional de Investigação em Psicologia, Faro, 30 de junho a 2 de julho (pp. 69). Revista de Psicologia da Associação Portuguesa de Psicologia. Lisboa.

Zar, J. H. (2010). Biostatistical Analysis (5th ed.). Prentice Hall.

Siegel S. Non-parametric statistics for the behavioral sciences. New York: McGraw-Hill,1956 \& 1988.

Swinscow TDV, Campbell MJ. Statistics at square one (10th ed). London: BMJ Books, 2002.

Mehta CR, Patel NR. A network algorithm for performing Fisher's exact test in rxc contingency tables. JASA, 1983; 78(382):427-434. 